Meta

Journal des traducteurs

Translators' Journal

\title{
Évaluation pédagogique de la recherche terminologique
}

\section{Lina Sader Feghali}

Volume 46, numéro 2, juin 2001

Évaluation : paramètres, méthodes, aspects pédagogiques /

Evaluation: Parameters, Methods, Pedagogical Aspects

URI : https://id.erudit.org/iderudit/002737ar

DOI : https://doi.org/10.7202/002737ar

Aller au sommaire du numéro

Éditeur(s)

Les Presses de l'Université de Montréal

ISSN

0026-0452 (imprimé)

1492-1421 (numérique)

Découvrir la revue

Citer cet article

Sader Feghali, L. (2001). Évaluation pédagogique de la recherche terminologique. Meta, 46(2), 426-437. https://doi.org/10.7202/002737ar

\section{Résumé de l'article}

Toute pratique pédagogique ne peut réussir sans mettre à l'épreuve les acquis des apprenants. Cette mise à l'épreuve requiert des instruments d'évaluation qui permettraient à l'enseignant, d'une part, de diagnostiquer les difficultés d'apprentissage et à l'apprenant, d'autre part, de comprendre ses erreurs et de les corriger. Cet article présente une stratégie d'évaluation suivie dans le cadre d'un cours d'initiation à la recherche documentaire et terminologique qui tente d'unir les deux approches sommative et formative pour aboutir à une évaluation objective des activités des apprenants. 


\title{
Évaluation pédagogique de la recherche terminologique
}

\author{
LINA SADER FEGHALI \\ Université Saint-Joseph, Beyrouth, Liban
}

\begin{abstract}
RÉSUMÉ
Toute pratique pédagogique ne peut réussir sans mettre à l'épreuve les acquis des apprenants. Cette mise à l'épreuve requiert des instruments d'évaluation qui permettraient à l'enseignant, d'une part, de diagnostiquer les difficultés d'apprentissage et à l'apprenant, d'autre part, de comprendre ses erreurs et de les corriger. Cet article présente une stratégie d'évaluation suivie dans le cadre d'un cours d'initiation à la recherche documentaire et terminologique qui tente d'unir les deux approches sommative et formative pour aboutir à une évaluation objective des activités des apprenants.
\end{abstract}

\begin{abstract}
A teaching method can only succeed when students are tested on what they learned. It can be done by using evaluation tools which enable the teacher to diagnose the learning difficulties and the students to better understand their mistakes and be able to correct them. This article presents an evaluation strategy that is used in the Introductory course to Documentary and Terminology Research which relies on both the summative and formative approaches to evaluate objectively the students work.
\end{abstract}

\section{MOTS-CLÉS/KEYWORDS}

terminologie, évaluation de la qualité, instruments d'évaluation, pédagogie de la terminologie, apprentissage

\section{Introduction}

La qualité en matière de terminologie demeure un sujet épineux qui fait l'objet de nombreux débats dans les cercles professionnels. Quoiqu'elle soit soumise à certaines normes internationales et que l' « on parle donc, et plus fréquemment encore ces derniers temps, de la qualité terminologique pour indiquer qu'une collection de termes spécialisés est de qualité dans la mesure où ces termes sont fiables, adéquats et corrects» (Cabré Castellvi 1998: 7), cette qualité demeure tributaire de trois sortes de contraintes. D'abord, des choix institutionnels ou individuels, quand il s'agit de défendre la terminologie propre à une entreprise ou à un auteur. Ensuite, des intérêts politiques qui favorisent une certaine terminologie dans le cadre d'un programme d'aménagement linguistique. Enfin, des contraintes budgétaires qui veillent à maintenir un rapport plus rentable entre la qualité de l'information terminologique et le capital investi. Par conséquent, il est difficile de trouver un modèle idéal de terminologie et des critères objectifs d'évaluation. Cependant, dans un contexte de formation, la réalité est tout autre: l'enseignant peut lui-même «créer» le modèle idéal en fonction des objectifs pédagogiques fixés et mettre ainsi au point une évaluation critériée pour juger objectivement une recherche documentaire et terminologique. 
Avant de présenter la stratégie d'évaluation suivie, ses instruments, ses avantages et ses limites, il convient de définir l'évaluation et de la placer dans un contexte pédagogique.

\section{Qu'est-ce qu' «évaluer» dans un contexte pédagogique?}

Dans son sens général, évaluer signifie:

- recueillir un ensemble d'informations suffisamment pertinentes, valides et fiables

- et examiner le degré d'adéquation entre cet ensemble d'informations et un ensemble de critères adéquats aux objectifs fixés au départ ou ajustés en cours de route

- en vue de prendre une décision. (De Ketele et Roegiers 1993:41)

Il est par conséquent nécessaire pour tout processus d'évaluation qui a toujours un caractère décisionnel de recourir à des critères précis pour comparer entre des attentes et des réalités. L'évaluateur serait alors, comme le décrit Charles Hadji (1992: 29), «en position d'intermédiaire entre le prescripteur, qui dit comment devrait être l'objet évalué [...] et l'observateur, qui dit comment est l'objet dans sa réalité concrète $[\ldots] »$. Mais cette activité de médiation entre des normes idéales et la réalité sur le terrain revêt des formes différentes selon la situation dans laquelle elle s'exerce. Dans un contexte pédagogique, il est nécessaire de mettre au point des démarches évaluatives efficaces et objectives qui reposent sur des objectifs clairement définis. En effet, pour réussir l'évaluation, il faut commencer par le premier maillon de la chaîne: la pratique pédagogique. Les objectifs spécifiques d'un cours étant intimement liés à l'évaluation, les instruments d'évaluation ne sont utiles, valides et pertinents que dans la mesure où ils permettent d'évaluer les performances par rapport à des objectifs préalablement établis. Si certains objectifs prédéfinis ne sont pas atteints, l'évaluation permettra un feed-back pour réexaminer les stratégies d'apprentissage.

Quant au recueil d'informations il se fait donc grâce à des instruments différents (exposés oraux, travaux pratiques, travaux théoriques, etc.) qui permettent de savoir ce qui a été fait et de le comparer avec ce qui doit être fait (les objectifs de l'exercice, les consignes, les principes théoriques expliqués dans le cadre du cours, etc.) pour aboutir enfin à une décision qui a souvent recours, dans un contexte pédagogique, à la mesure. En effet, en fin de parcours, il ne suffit pas à l'enseignant de diagnostiquer les difficultés d'apprentissage et de vérifier l'acquisition d'une compétence. De même, l'apprenant ne doit pas seulement comprendre ses erreurs et les corriger, il faut aussi que cette évaluation aboutisse à une note qui sanctionne ces travaux et donne une appréciation quantitative qui situe l'apprenant par rapport aux autres.

Quoique la mesure ne soit pas le propre de l'évaluation, il est impossible d'envisager cette dernière dans un contexte académique sans parler de mesure. En effet, il est nécessaire de traduire une manifestation comportementale en système numérique à des fins de comparaison, de précision, de rigueur et de standardisation. Cependant, le quantitatif n'a de valeur que dans la mesure où il se met au service du qualitatif. Ainsi, le barème est établi en fonction de l'importance relative de la compétence visée par l'instrument d'évaluation qui sanctionne les fautes graves plus sévèrement que les fautes mineures et qui prend en considération la prestation de tout un groupe.

Ainsi, l'évaluation sert en général à sanctionner les apprentissages. L'évaluation 
pédagogique se fait en fonction des objectifs d'apprentissage fixés par l'enseignant et selon un barème qui sanctionne les fautes graves plus sévèrement que les fautes mineures et qui prend en considération la prestation de tout un groupe.

\section{Quelle stratégie d'évaluation suivre?}

Ces considérations d'ordre général s'appliquent donc à tout cours de formation académique. Cependant, la stratégie d'évaluation varie selon les objectifs de chaque cours. La stratégie proposée dans le cadre de cet article est celle qui permet d'évaluer des apprenants qui suivent un cours d'initiation à la méthodologie de recherche documentaire et terminologique ${ }^{1}$.

Cette stratégie s'inscrit dans le cadre de la pédagogie par les objectifs et intervient à toutes les étapes de l'apprentissage pour savoir si le cours a porté ses fruits. C'est la taxonomie des objectifs cognitifs de Bloom qui a aidé à déterminer les objectifs d'apprentissage ${ }^{2}$, car il est inconcevable de prévoir un instrument d'évaluation sans savoir au juste quel est le type de savoir-faire qui fait l'objet de cette évaluation. À titre illustratif, nous proposons pour chaque niveau de la taxonomie des objectifs d'apprentissage du domaine cognitif de Bloom un objectif d'apprentissage et un instrument d'évaluation prévu dans le cadre de la formation à la recherche documentaire et terminologique:

\begin{tabular}{|c|c|c|}
\hline Niveau & Objectif & Instrument d'évaluation \\
\hline $\begin{array}{l}\text { 1. Acquisition } \\
\text { de connaissances }\end{array}$ & $\begin{array}{l}\text { - Distinguer entre lexicologie, } \\
\text { lexicographie/terminologie, } \\
\text { terminographie, terminotique; }\end{array}$ & $\begin{array}{l}\text { - Travail de groupe en classe: } \\
\text { relever dans un tableau synoptique les } \\
\text { différences entre ces disciplines. }\end{array}$ \\
\hline 2. Compréhension & $\begin{array}{l}\text { - Décrire un texte spécialisé } \\
\text { - Comparer entre une entrée } \\
\text { lexicographique et une entrée } \\
\text { terminologique }\end{array}$ & $\begin{array}{l}\text { - Travail de groupe en classe: relever } \\
\text { dans un texte spécialisé les caractéristiques } \\
\text { des langues de spécialité. } \\
\text { - Travail de groupe en classe: reconnaître } \\
\text { une entrée terminologique en la } \\
\text { comparant à une entrée lexicographique } \\
\text { ou relever les lacunes de cette dernière. }\end{array}$ \\
\hline 3. Application & $\begin{array}{l}\text { - Manipuler les outils de } \\
\text { recherche documentaire } \\
\text { et terminologique }\end{array}$ & $\begin{array}{l}\text { - Travail individuel ou en groupe hors } \\
\text { des heures de cours: relever les termes } \\
\text { propres au domaine choisi selon } \\
\text { des critères précis (par exemple, critères } \\
\text { documentaire de fréquence et de } \\
\text { répartition, critères linguistiques). } \\
\text { - Travail individuel ou en groupe hors } \\
\text { des heures de cours: dresser un inventaire } \\
\text { des ressources lexicographiques et non } \\
\text { lexicographiques d'un domaine précis } \\
\text { à travers l'interrogation de banques } \\
\text { de données terminologiques } \\
\text { et documentaires, la consultation } \\
\text { de bibliothèques, etc. }\end{array}$ \\
\hline 4. Analyse & $\begin{array}{l}\text { - Élaborer le réseau notionnel } \\
\text { du sous-domaine choisi. }\end{array}$ & $\begin{array}{l}\text { - Travail individuel ou en groupe hors } \\
\text { des heures de cours: savoir identifier } \\
\text { les rapports existant entre les différentes } \\
\text { notions (logiques, ontologiques). }\end{array}$ \\
\hline
\end{tabular}




\begin{tabular}{|c|c|c|}
\hline 5. Synthèse & $\begin{array}{l}\text { - Exposer oralement la } \\
\text { terminologie de base } \\
\text { du sous-domaine étudié. } \\
\text { - Produire des dossiers } \\
\text { et des fiches terminologiques. } \\
\text { - Discuter une observation } \\
\text { d'ordre terminologique. }\end{array}$ & $\begin{array}{l}\text { - Présentation en classe: vérifier } \\
\text { l'adaptation de l'exposé aux destinataires } \\
\text { et l'acquisition des notions de base } \\
\text { du sous-domaine. } \\
\text { - Travail individuel ou en groupe hors des } \\
\text { heures de cours: évaluer la capacité } \\
\text { d'exploiter les ressources documentaires } \\
\text { à des fins terminologiques pour élaborer } \\
\text { la définition, choisir le contexte pertinent, } \\
\text { relever une remarque d'ordre technique } \\
\text { ou linguistique, etc. (voir annexe 2). } \\
\text { - Travail individuel ou en groupe hors des } \\
\text { heures de cours: évaluer la capacité } \\
\text { de l'apprenant de relever une particularité } \\
\text { terminologique du sous-domaine étudié } \\
\text { par l'agencement de plusieurs éléments } \\
\text { en une structure originale et personnelle. }\end{array}$ \\
\hline 6. Évaluation & $\begin{array}{l}\text { - Évaluer les sources } \\
\text { lexicographiques et } \\
\text { non lexicographiques. }\end{array}$ & $\begin{array}{l}\text { - Travail individuel ou en groupe hors des } \\
\text { heures de cours: s'assurer de la conformité } \\
\text { de l'évaluation aux critères prédéfinis. }\end{array}$ \\
\hline
\end{tabular}

Cependant, même avec des objectifs bien définis, il est impossible de planifier une stratégie d'évaluation sans cerner le cadre de l'évaluation: pourquoi évalue-ton? Pour qui évalue-t-on? Quand évaluer? En effet, l'évaluation n'est pas un but en soi: elle est en général déterminée en fonction du type de décision à prendre et de l'objet de l'évaluation, ces deux éléments qui, à leur tour, aident à déterminer le moment de l'évaluation.

Ainsi, toute évaluation doit prendre en considération les trois principales fonctions de l'évaluation: pronostic, diagnostic, inventaire (Tagliante 1991: 14).

L' «évaluation-pronostic» — grâce à laquelle l'enseignant vérifie les prérequis d'un apprentissage et en analyse les besoins - a lieu, dans ce genre de cours, suite à une mise au point au début de l'apprentissage qui permet à l'apprenant d'adapter les objectifs de formation aux besoins et au niveau du groupe. Ainsi, au début de chaque année universitaire, l'enseignant invite chaque apprenant à faire le point sur ses acquis et ses prérequis (en linguistique et en lexicographie) ainsi que sur ses attentes et ses motivations après la lecture du plan du cours en question. Cette évaluation-pronostic constitue une source d'information essentielle pour relever les points faibles et les points forts du groupe en général et de chaque apprenant en particulier.

L'«évaluation-diagnostic» qui intervient tout au long du cursus de formation, vise à à corriger ou à ajuster le processus de formation en vue de l'améliorer. Elle est à la fois formative et formatrice. Formative parce qu'elle est mise au service de l'apprenant lui permettant d'analyser ses erreurs et d'améliorer sa progression. Formatrice parce qu'elle est mise au service du formateur lui permettant de revoir ses stratégies d'enseignement. Cette évaluation de régulation a lieu grâce aux travaux sur table et notamment aux mini-rapports de recherche (voir Annexe 1) que les apprenants présentent pour faire état de l'avancement de leurs travaux, faire part de leurs problèmes auxquels une solution est trouvée avec l'enseignant. Ils y annexent aussi les travaux accomplis (inventaire bibliographique, dossiers terminologiques, réseau notionnel, etc.) qui seront évalués par l'enseignant puis corrigés par l'apprenant 
avant d'être insérés dans le dossier final. Cette évaluation conçue dans un esprit formatif est critériée (Tagliante 1991: 18), c'est-à-dire que l'apprenant ou le groupe n'est pas comparé aux autres mais, c'est en référence à des critères qu'il est possible de vérifier que l'enseignant juge si l'apprenant maîtrise tel objectif et est en mesure de passer à d'autres apprentissages.

C'est à ce moment que l'enseignant a conscience de l'intérêt de l'évaluationdiagnostic qui permet une approche plus personnalisée de l'évaluation, car chaque domaine de recherche présente une difficulté particulière qui requiert des stratégies différentes. Ainsi, cette vérification, étape par étape, permet à l'apprenant de prendre conscience de ce qui lui reste à faire tout en permettant à l'enseignant de repérer les failles et de réguler ses pratiques pédagogiques.

Ainsi, avec ces deux évaluations, l'apprenant repère les lacunes qui l'empêchent d'atteindre les objectifs d'apprentissage. Il apprend à son propre rythme et décide de son propre cheminement. Il est responsable de son apprentissage, car l'enseignant est là pour le guider et l'orienter et non pour le sanctionner par une note. En effet, les mini-rapports de recherche permettent à l'enseignant de suivre de près le travail des apprenants pour garantir la qualité du travail final qui fera l'objet d'une évaluationinventaire.

L' «évaluation-inventaire» est celle qui est exprimée en termes de réussite et d'échec. Intervenant en fin de cursus, elle dresse le bilan des acquis et certifie qu'un certain niveau est atteint. C'est une évaluation sommative qui dresse l'inventaire des savoirs et des savoir-faire de l'apprenant (dossier de recherche final et fiches terminologiques) grâce à une grille dans laquelle l'enseignant établit les critères d'appréciation et de mesure. (voir annexes 2 et 3 ).

Cette stratégie d'évaluation qui prend en considération les fonctions de l'évaluation et qui intervient à toutes les étapes du processus, c'est-à-dire qui ne se limite pas à l'étape finale «quand il est déjà trop tard», permet une vision d'ensemble de l'apprentissage. Ainsi entre l'évaluation-pronostic qui s'inscrit dans une perspective individuelle (est-ce que l'apprenant pourra atteindre les objectifs visés dans le contexte existant ou des changements sont-ils nécessaires?) et l'évaluation de certification qui s'inscrit dans une perspective sociale (est-ce que cet apprenant réussira et répond au profil attendu?), l'évaluation de régulation porte sur des problèmes de fonctionnement et se situe entre la dimension individuelle et la dimension sociale (que faut-il faire pour atteindre les objectifs dans les meilleures conditions?).

\section{Avec quels outils d'évaluation?}

L’objectif général du cours étant d'initier les apprenants à l'activité documentaire et terminologique qui intervient dans le processus traductionnel, il suppose l'acquisition de plusieurs savoirs et savoir-faire et a recours à divers outils d'évaluation.

D'abord, les mini-rapports de recherche revêtent une importance particulière dans le cadre de ce cours puisqu'ils permettent aux apprenants de s'assurer de la démarche suivie et d'ajuster le tir. Ces mini-rapports ne sont pas notés mais sont pris en considération dans la notation finale et font l'objet de notes supplémentaires qui récompensent la régularité du travail.

Autre outil d'évaluation, l'évaluation faite suite à un exposé oral au cours duquel l'apprenant ou l'équipe constituée 3 présente la terminologie de base du sous-domaine 
étudié dans un aperçu qui s'adapte aux destinataires (des apprentis-traducteurs). Cet exposé comprend trois temps: dans un premier temps, un QCM tente d'évaluer le niveau de connaissances de la classe. Dans un deuxième temps, le QCM est corrigé et des informations plus détaillées sont présentées. Dans un troisième temps, un court questionnaire sert à vérifier l'acquisition des notions présentées. La notation de cet exposé prend en considération ces trois temps selon un barème bien précis. Cet exposé, malgré son apparence sommative, permet à l'enseignant de relever les points de faiblesse ou les lacunes qui risqueraient de compromettre les résultats du travail final.

Quant au dossier de recherche final qui comprend les fiches terminologiques, il fait l'objet de grilles d'évaluation qui prennent la forme d'une évaluation sommative qui sanctionne en fin d'année la totalité des travaux entrepris.

Compte tenu du fait que ce sont ces grilles qui permettent d'évaluer la totalité des acquis et qu'il est impossible, dans le cadre de cet article, de décrire tous les outils utilisés, nous nous limiterons aux deux grilles d'évaluation qui sont mises au point pour permettre à l'enseignant de vérifier la présence de ce qui est attendu en fin d'apprentissage, à savoir les compétences à acquérir (voir annexes 2 et 3 ).

Ces grilles d'évaluation se présentent sous la forme d'une série de questions classées en catégories. Dans la grille d'évaluation du dossier final, deux grandes catégories sont prévues: la forme et le contenu. Quoique ne constituant que $20 \%$ de la note finale, l'appréciation de la forme vérifie la capacité de l'apprenant à se conformer à des règles de présentation bien établies ainsi qu'au calendrier fixé. Il ne faut pas oublier que ce cours est destiné à de futurs traducteurs et interprètes; c'est-à-dire à de futurs professionnels qui seront jugés sur la présentation formelle de leur travail et sur le respect des délais.

Dans la partie consacrée à l'appréciation du contenu, l'enseignant évalue chacune des parties du dossier final qui ne sont qu'une synthèse de la recherche entreprise en cours d'année. Les questions qui y figurent permettent de vérifier l'acquisition de plusieurs savoirs et savoir-faire, donc plusieurs objectifs spécifiques à la fois. Prenons par exemple les deux questions suivantes:

La méthode de recherche documentaire est-elle décrite (évaluation des sources)?

La bibliographie est-elle diversifiée, complète, fiable et pertinente?

À travers ces deux questions et l'évaluation des annexes supplémentaires fournies par l'apprenant (description d'un site Internet, d'un ouvrage ou d'un centre de documentation), c'est surtout l'acquisition d'une capacité méthodologique qui est mise à l'épreuve. Dans un premier temps, l'apprenant est appelé à rassembler le maximum de documents portant sur le domaine de la recherche dans toutes les langues de travail et dans toutes les catégories et à trier ces documents pour n'en retenir que les plus pertinents et sélectionner les parties qui y sont les plus utiles pour la recherche.

La constitution du corpus de dépouillement se fait en fonction de critères bien précis: fiabilité, pertinence et variété des ouvrages à consulter sans oublier l'importance à accorder à la date et au lieu de publication; les ouvrages récents et dont l'usage ne se limite pas à une région géographique déterminée étant les plus privilégiés. Les sources consultées ne sont pas toutes à support papier et compte tenu du contraste entre l'innovation lexicale et le rythme de la progression de l'information documentaire, l'Internet et les experts sont aussi une source d'information à prendre en considération. Dans un deuxième temps, l'apprenant doit établir, au fur et à mesure 
de l'avancement de la recherche documentaire, une liste bibliographique complète des documents consultés. En effet, c'est grâce à cette liste, qu'il est possible à l'enseignant de vérifier la cohérence, le sérieux et la complétude de la recherche. Enfin, dans un troisième temps, l'apprenant est appelé à évaluer les documents en termes de lisibilité et de fiabilité en fonction des objectifs de la recherche documentaire, à savoir:

Se familiariser avec le sujet de la recherche et/ou acquérir des informations complémentaires;

Fournir le matériel de travail (corpus) nécessaire pour une recherche terminologique exhaustive, fiable et précise.

Un suivi régulier de cette étape fondamentale, grâce notamment aux minirapports de recherche, contribue à la réussite de la présentation de cette étape de la recherche mais, dans son évaluation finale, l'enseignant prend aussi en considération l'accessibilité de la source, le temps dont disposent les apprenants effectivement, les coûts et les efforts (déplacements, attentes, contacts désagréables, etc.). C'est donc une évaluation par critère qui est mise en application, car chaque domaine de recherche a ses difficultés documentaires propres. Ainsi, pour une recherche terminologique dans un domaine de pointe, il est difficile de trouver des ouvrages lexicographiques ainsi que des ouvrages de référence et ce sont souvent les supports informatiques (Internet et CD-ROM) et la littérature grise, c'est-à-dire la documentation non publiée par la voie de l'édition classique, qui peuvent servir de référence. Par contre, si l'apprenant choisit de travailler sur la terminologie d'un domaine qui ne subit plus relativement de modifications comme, à titre d'exemple, tout ce qui touche aux arts classiques (arabesque, antiquités, etc.), il est souvent obligé de retenir des ouvrages qui gardent leur valeur malgré leur année d'édition assez ancienne.

Quant à la capacité de l'apprenant de mener à bien une recherche terminologique et de la décrire, elle est évaluée grâce aux questions suivantes (les dossiers terminologiques et les fiches terminologiques faisant l'objet d'une grille d'évaluation séparée) :

Le sujet de recherche est-il clairement présenté en fonction de son intérêt terminologique?

Le sous-domaine a-t-il été logiquement cerné?

Lélaboration du réseau notionnel a-t-elle été décrite?

Les critères de repérage des termes ont-t-ils été présentés?

La méthode d'élaboration des fiches est-elle décrite?

L'arbre du domaine et le réseau notionnel sont-ils conformes aux normes?

L'article présente-t-il une trouvaille terminologique intéressante? (sujet bien cerné et bien documenté )

Les termes figurant dans le lexique sont-ils propres au sous-domaine traité?

Une définition précise dans l'une des langues de travail a-t-elle été incluse dans le lexique?

Dans cet effort de synthèse, l'apprenant doit être capable d'exploiter le cours pour décrire sa recherche. Toute référence aux principes et méthodes présentés par l'enseignant et appliqués à la recherche contribue à une évaluation positive du travail. À titre d'exemple, l'enseignant s'attend à ce que l'apprenant utilise la terminologie appropriée lors de la description du travail entrepris: il doit montrer qu'il est capable de discerner entre un contexte définitoire, un contexte explicatif et un contexte associatif. Il doit aussi savoir préciser le type de définition adoptée (définition 
générique, partitive, lexicale, etc.) justifiant les raisons de telle définition ou telle autre. Il faut noter aussi que certains choix et travaux (choix du sous-domaine, élaboration de l'arbre du domaine et du réseau notionnel et trouvaille terminologique ${ }^{4}$ ) ont fait l'objet d'une évaluation-diagnostic dans le cadre des mini-rapports de recherche.

Par ailleurs, une grille séparée évalue la capacité de l'apprenant de constituer des dossiers terminologiques et d'élaborer des fiches terminologiques. La recherche terminologique portant sur un sous-domaine précis ne retient que les termes qui appartiennent au sous-domaine en question. L'objectif étant d'apprendre à exploiter l'information documentaire à des fins terminologiques, ce sont les termes les plus fréquents du domaine qui font l'objet d'une recherche terminologique approfondie. Les langues de départ et d'arrivée sont aussi choisies en fonction de la disponibilité de la documentation. Autrement dit, la recherche faite dans les meilleures conditions possibles dans un premier temps permet aux apprenants d'avoir conscience de l'importance de l'approche sans faire face immédiatement aux frustrations de l'absence de l'information ou de son insuffisance. Une fois les données rassemblées, il est possible de cerner les notions et les termes qui les désignent, d'établir l'équivalence notion à notion et terme à terme et aussi de constater en simples observateurs les particularités des termes étudiés.

Enfin, c'est le plus pertinent qui est consigné dans des fiches terminologiques mises au point dans le but d'initier les apprenants à la pratique terminographique. Pour évaluer la pertinence du choix de l'apprenant dans chacun des blocs de la fiche, les principes à suivre impérativement sont mis sous forme de questions (voir annexe 3). Cependant, l'évaluation est «critériée », c'est-à-dire qu'elle est faite en fonction du dossier terminologique constitué et de la lisibilité de la fiche. À noter que les apprenants ont la possibilité, en cours d'année, de vérifier l'acquisition de la pratique terminographique en demandant à l'enseignant de corriger une fiche-prototype qui leur servirait de modèle à suivre pour l'élaboration des quatre restantes. Ainsi, l'enseignant attire leur attention sur les critères pris en considération lors de l'évaluation finale leur permettant d'éviter les fautes graves.

Une description de ces grilles serait incomplète sans le barème de notation. En effet, toute évaluation dans un contexte pédagogique suppose des résultats chiffrés. C'est pour cela que les grilles d'évaluation sont accompagnées d'un barème qui reflète l'importance relative de chaque critère d'appréciation. Ce barème très souple avantage l'apprenant car il prévoit une note moyenne ( presque») pour une performance imparfaite.

\section{Conclusion}

Bonne ou mauvaise recherche documentaire et terminologique sont des qualificatifs qu'il serait difficile de définir avec précision et dans l'absolu en l'absence d'objectifs d'apprentissage et d'outils d'évaluation. La mise au point de grilles contribue à éviter toute subjectivité de la part de l'enseignant et aide l'apprenant à comprendre les raisons de sa réussite ou de son échec. Cependant, malgré toutes les tentatives d'assurer un suivi périodique des activités des apprenants, cette stratégie d'évaluation demeure tributaire de la bonne volonté des apprenants de rendre compte de l'avancement de leur recherche dans les mini-rapports de recherche car, ces derniers 
n'étant pas notés, ils ne sont pas toujours pris au sérieux et ainsi l'apprenant perd l'occasion qui lui est offerte de bénéficier d'une évaluation formative et formatrice. Il se trouve alors confronté à une évaluation sommative qui intervient en fin d'année pour sanctionner une série d'activités entreprises tout au long de l'année. Ces grilles, quoiqu'elles facilitent la correction, ressemblent davantage à une check-list pour vérifier la complétude du travail. En effet, pour répondre aux questions de cette grille de type ouvert, l'enseignant essaie d'apprécier la performance de l'apprenant en fonction de deux critères: la mise en application des principes et méthodes transmises dans le cadre du cours et la touche personnelle de l'apprenant. En effet, «évaluer une compétence complexe, c'est prendre en compte non seulement les divers savoirs et savoir-faire dont elle est constituée, mais aussi tout ce qui relève de la sensibilité, de l'imagination, de l'opinion personnelle et de l'affectivité de l'élève. En un mot, de sa personnalité. Dès que l'on quitte l'évaluation des connaissances élémentaires, la mesure se transforme en appréciation» (Tagliante 1991:30).

En définitive, pour réussir toute évaluation, il faut que les objectifs d'apprentissage soient clairs pour les deux parties impliquées dans l'apprentissage, car l'évaluation permet un feed-back et par conséquent une évolution permanente du cours; si le message ne passe pas, c'est-à-dire si tout le groupe n'a pas réussi à atteindre un objectif d'apprentissage, l'enseignant sera conscient de la nécessité de réexaminer les stratégies d'apprentissage. Par ailleurs, en dévoilant ces critères d'évaluation, donc en adoptant une attitude de transparence, à travers une évaluation formative "critériée ", l'enseignant n'est plus le seul juge menaçant des performances mais il offre à l'apprenant l'occasion de s'autoévaluer et de constater l'écart entre le résultat escompté et le résultat tangible.

Quoi qu'il en soit, les outils utilisés ne doivent pas être considérés comme une panacée qui résout tous les problèmes d'évaluation ni comme une structure rigide qui n'accepte aucune modification. Ils ne sont que de simples indicateurs qui donnent l'alerte quand la pratique pédagogique n'atteint pas ses objectifs.

\section{NOTES}

1. Ce sont des étudiants en troisième année à l'école de traducteurs et d'interprètes de Beyrouth. Ce cours fait suite à des cours d'initiation à la linguistique générale en première année et à des cours d'initiation à la sémantique, à la lexicologie et la lexicographie en deuxième année. Ce cours est complété en quatrième année, pour les traducteurs seulement, par un cours consacré aux problèmes terminologiques.

2. Il convient de rappeler que ces objectifs tentent de répondre aux besoins de formation de futurs traducteurs et interprètes et que ce cours ne vise pas à former des terminologues.

3. Les étudiants peuvent choisir entre le travail individuel et le travail d'équipe.

4. Cerner une notion; relever un cas de polysémie; constater l'influence d'une langue dans un domaine précis qui aboutit à un phénomène d'emprunt; répertorier les synonymes d'un terme.

\section{RÉFÉRENCES}

Cabré Castellvi, M. T. (1998): «À propos de la notion de qualité en terminologie», La banque des mots, 8 , numéro spécial, p. 7-34.

De Ketele, J.-M. et X. Roegiers (1993): Méthodologie du recueil d'informations: fondements des méthodes d'observations, de questionnaires, d'interviews et d'études de documents, Bruxelles, Éditions Université de Boeck.

HADJI, C. (1992): L'évaluation, règles du jeu: des intentions aux outils, Paris, ESF. 
Prégent, R. (1990): La préparation d'un cours, Montréal, Éditions de l'école polytechnique de Montréal.

Tagliante, C. (1991): L'évaluation, Paris, Clé International.

\section{ANNEXES}

\section{Mini-rapport de recherche (4)}

- Prénoms et NOM:

- Domaine et sous-domaine choisis: (Arbre du domaine - version finale - en annexe).

- Réseau notionnel: (À fournir en annexe).

- Cinq termes retenus:

- Contextes relevés lors du dépouillement (à fournir en annexe les dossiers terminologiques en surlignant dans chaque contexte les traits sémantiques jugés pertinents).

N.B. Ne pas oublier de coder chaque référence!

- État de la recherche documentaire: (À fournir en annexe la liste bibliographique actualisée)

Ne pas oublier qu'il est nécessaire d'avoir accès à au moins deux ouvrages en LA.

- Proposez le sujet qui pourrait faire l'objet de l'article que vous consacrerez à une (des) trouvaille(s) terminologique(s) faite(s) en cours de recherche.

- Problèmes à signaler :

2. Grille d'évaluation du dossier

Notes supplémentaires sur la régularité du travail (présentation régulière des mini-rapports de recherche)

1. APPRÉCIATION DE LA FORME (/20)

1.1. Dans la mise en page ( /6)

Y-a-t-il des interlignes? oui $1 / 2-$ non 0

Y-a-t-il des marges acceptables et des retraits? oui $1-$ non 0

Y-a-t-il des coupures dans les mots, des orphelins à la fin des pages? oui 0 - non 1

Le numéro de la page (folio) est-il signalé? oui $1-$ non 0

Les procédés typographiques sont-ils utilisés? oui $1 \frac{112}{2}$ - non 0 - presque $3 / 4$

La présentation globale est-elle nette et cohérente? oui 1 - non 0 - presque $3 / 4$

Les termes dans le texte choisi sont-ils mis en évidence? oui $1 / 2-$ non 0

1.2. Dans la page-titre (/1)

La page-titre comporte-t-elle les informations nécessaires? oui 1 - non 0 - presque $1 / 2$

Est-elle attrayante, originale et bien disposée? oui $1 / 2-$ non 0

1.3. Dans la table des matières (/2)

Est-elle complète et fait-elle référence aux pages? oui 2 - non 0 - presque 1

Figure-t-elle au début du dossier? oui $1 / 2-$ non 0

1.4. Dans la bibliographie ( /6)

Est-elle subdivisée en catégories? oui 1 - non 0

Chaque référence est-elle précédée d'un numéro puis d'un code et enfin du lieu? oui 2 - non 0

Les références sont-elles présentées selon les normes? oui 3 - non 0 - presque 2 
1.5. Dans les annexes ( /2)

Sont-elles intitulées? oui 1 - non 0

Les références utilisées pour les annexes supplémentaires sont-elles signalées? oui 1 - non 0

1.6. Respect du calendrier ( /2)

Le dossier a-t-il été remis à la date fixée? oui 2 - retard de $24 \mathrm{~h} 0--2$ points par jour de retard

2. APPRÉCIATION DU CONTENU ( /80)

2.1. Dans la globalité du dossier ( /10)

Le style est-il correct? oui 6 - non 0 - presque $2^{1 / 2}$

L'orthographe et la ponctuation sont-elles correctes? oui 4 - non 0 - presque 3

2.2. Dans la présentation du travail de recherche (/36)

Le sujet de recherche est-il clairement présenté en fonction de son intérêt terminologique? oui 3 - non 0 - presque $1^{1 / 2}$

La méthode de recherche documentaire a-t-elle été décrite (évaluation des sources)? oui 10 - non 0 - presque 5

Le sous-domaine a-t-il été logiquement cerné? oui 3 - non 0 - presque $1 \frac{1 / 2}{2}$

L'élaboration du réseau notionnel a-t-elle été décrite? oui 6 - non 0 - presque 3

Les critères de repérage des termes ont-t-ils été présentés? oui 3 - non 0 - presque $1 \frac{1 / 2}{2}$

La méthode d'élaboration des fiches est-elle présentée? oui 6 - non 0 - presque 3

Le travail entrepris a-t-il été évalué en fonction des objectifs spécifiques du cours? oui 1 - non 0 - presque $1 \frac{1}{2}$

La bibliographie est-elle diversifiée, complète, fiable et pertinente? oui 4 - non 0 - presque 2

2.3. Dans les annexes obligatoires ( /20)

Le dossier comporte-t-il les annexes obligatoires? oui $1-$ non 0 - presque $1 / 2$

L'arbre du domaine et le réseau notionnel sont-ils conformes aux normes? oui 7 - non 0 - presque $3 \frac{1}{2}$

Le dossier comporte-t-il des annexes qui le rendent original et qui complètent le dossier? oui 2 - non 0 - presque 1

L'article présente-t-il une trouvaille terminologique intéressante? (sujet bien cerné et bien documenté ) oui 10 - non 0 - presque 5

2.4. Dans les annexes supplémentaires ( /14)

Le site Internet est-il bien choisi et bien décrit? oui 7 - non 0 - presque $31 / 2$

Les informations relatives aux dictionnaires ou ouvrages à recommander sont-elles pertinentes et complètes? oui 7 - non 0 - presque $3 \frac{11}{2}$

Les termes figurant dans le lexique sont-ils propres au sous-domaine traité? oui $3 \frac{1}{2}-$ non 0 - presque 2

Une définition précise dans l'une des langues de travail a-t-elle été incluse dans le lexique? oui $3 \frac{1}{2}-$ non 0 - presque 2

Les informations relatives au centre de documentation sont-elles pertinentes et complètes? oui 7 - non 0 - presque $3 \frac{1}{2}$

\section{3. ÉVALUATION DES FICHES TERMINOLOGIQUES}

Présentation générale: ( /2)

1. Dans le Bloc Gestion (/3)

Les noms, les initiales et la date de rédaction sont-ils signalés? oui $1-$ non 0

Le domaine générique est-il celui de la recherche? oui 1 - non 0

Le domaine spécifique est-il celui des termes-vedettes? oui $1-$ non 0 
2. Dans le Bloc Terminologie L1 ( /38)

Les termes choisis pour la vedette représentent-ils la forme dominante et attestée de la notion étudiée? oui 1 - non 0 - presque $1 / 2$.

Les indications grammaticales sont-elles signalées? oui $1 / 2-$ non 0

Les définitions sont-elles terminologiques? /30

\begin{tabular}{|l|l|l|l|l|l|}
\hline Contient les descripteurs qui: $(6$ points/fiche) & F1 & F2 & F3 & F4 & F5 \\
\hline permettent de distinguer la notion étudiée des notions apparentées $(1)$ & & & & & \\
\hline décrivent de façon précise le sens des termes définis $\left(2 \frac{1}{2}\right)$ & & & & & \\
\hline sont complets mais sans excès $\left(2 \frac{1}{2}\right.$ pts) & & & & & \\
\hline
\end{tabular}

Les contextes complètent-ils la définition et/ou mettent-ils en évidence des collocations? oui 3 - non 0 - presque $1 \frac{1 / 2}{2}$

Les notes (techniques et/ou linguistiques) sont-elles pertinentes? oui 3 - non 0 - presque $1 \frac{1 / 2}{2}$

Les synonymes (s'il y en a) sont-ils des cas de vraie synonymie? oui 2 - non 0 - presque 1

3. Dans le Bloc Terminologie L2 ( /38)

Les termes choisis pour la vedette représentent-ils la forme dominante et attestée de la notion étudiée? oui 1 - non 0 - presque $1 / 2$.

Les indications grammaticales sont-elles signalées? oui $1 / 2-$ non 0

Les définitions sont-elles terminologiques? /30

\begin{tabular}{|l|l|l|l|l|l|}
\hline Contient les descripteurs qui: $(6$ points/fiche) & F1 & F2 & F3 & F4 & F5 \\
\hline fournissent un crochet terminologique solide $(1)$ & & & & & \\
\hline décrivent de façon précise le sens des termes définis $\left(2 \frac{1}{2}\right)$ & & & & & \\
\hline sont complets mais sans excès $\left(2 \frac{1}{2}\right)$ & & & & & \\
\hline
\end{tabular}

Les contextes complètent-ils la définition et/ou mettent-ils en évidence des collocations? oui 3 - non 0 - presque $1 \frac{1 / 2}{1}$

Les notes (techniques et/ou linguistiques) sont-elles pertinentes? oui 3 - non $0-$ presque $1^{1 / 2}$

Les synonymes (s'il y en a) sont-ils des cas de vraie synonymie? oui 2 - non 0 - presque 1

4. Dans le Bloc Sources ( /18)

Les renvois aux sources sont-ils méthodiques et les sources sont-elles diversifiées? oui 5 - non 0 - presque $2^{1 / 2}$

Les dossiers terminologiques sont-ils pertinents et complets? oui 10 - non 0 - presque 5

Le repérage est-il méthodique? oui 3 - non 0 - presque $1^{1 / 2}$ 